Pacific

Journal of

Mathematics

TRANSLATING SOLUTIONS FOR GAUSSCURVATURE FLOWS WITH NEUMANN BOUNDARY CONDITIONS 


\section{TRANSLATING SOLUTIONS FOR GAUSSCURVATURE FLOWS WITH NEUMANN BOUNDARY CONDITIONS}

We consider strictly convex hypersurfaces which are evolving by the non-parametric logarithmic Gauß curvature flow subject to a Neumann boundary condition. Solutions are shown to converge smoothly to hypersurfaces moving by translation. In particular, for bounded domains we prove that convex functions with prescribed normal derivative satisfy a uniform oscillation estimate.

\section{Introduction.}

In this paper, we evolve hypersurfaces represented as graphs of strictly convex functions over strictly convex bounded domains by the non-parametric logarithmic Gauß curvature flow subject to a Neumann boundary condition. We show that solutions exist for all time and converge smoothly to translating solutions.

To be more precise, we address the following slightly more general problem: Let $u_{0}$ be a strictly convex function over a smooth strictly convex bounded domain $\Omega \subset \mathbb{R}^{n}$. We use the phrase strictly convex for functions whose Hessian is positive definite, and for domains for which all principal curvatures of the boundary are positive. Assume that $u_{0}$ is smooth up to the boundary, $u_{0} \in C^{\infty}(\bar{\Omega})$, and satisfies

$$
D_{\nu} u_{0}=\varphi \quad \text { on } \partial \Omega
$$

where $\nu$ is the inner unit normal to $\partial \Omega$ and $\varphi \in C^{\infty}(\partial \Omega)$. Let $f \in$ $C^{\infty}\left(\bar{\Omega} \times \mathbb{R}^{n}\right)$. We prove the following:

Theorem 1.1. For $\Omega, \varphi, \nu, f$ and $u_{0}$ as introduced above, there exists a family $u(\cdot, t), t \in[0, \infty)$, of strictly convex functions solving

$$
\left\{\begin{aligned}
\frac{\partial}{\partial t} u & =\log \operatorname{det} D^{2} u-\log f(x, D u) & & \text { in } \Omega \times[0, \infty), \\
D_{\nu} u(\cdot, t) & =\varphi & & \text { on } \partial \Omega, t>0, \\
u(\cdot, 0) & =u_{0} & & \text { in } \Omega,
\end{aligned}\right.
$$

where $u \in C^{\infty}(\bar{\Omega} \times(0, \infty))$, and $u(\cdot, t)$ approaches $u_{0}$ in $C^{2}(\bar{\Omega})$ as $t \rightarrow$ 0 . Moreover, $u(\cdot, t)$ converges smoothly to a translating solution, i.e., to a solution with constant time derivative.

We remark that the parabolic maximum principle implies that the asymptotic solutions for different initial data $u_{0}$ are unique up to a constant. 
For the Gauß curvature flow, mentioned in the beginning, the flow equation takes the form

$$
\frac{\partial}{\partial t} u=\log \frac{\operatorname{det} D^{2} u}{\left(1+|D u|^{2}\right)^{\frac{n+2}{2}}}=\log \operatorname{det} D^{2} u-\frac{n+2}{2} \log \left(1+|D u|^{2}\right) .
$$

In the proof of Theorem 1.1, we generalize the result to the so-called oblique boundary condition

$$
D_{\beta} u=\varphi \quad \text { on } \partial \Omega,
$$

where $\beta$ is a unit vector field which is $C^{1}$-close to $\nu$, i.e., such that there exists a small positive constant $c_{\beta}>0$ for which $\|\nu-\beta\|_{C^{1}} \leq c_{\beta}$. Such a generalization of a Neumann boundary condition is studied for the elliptic case in $[7]$.

We base the barrier construction in the proof of Theorem 1.1 on solutions to a related elliptic problem given by the following:

Theorem 1.2. Consider $\Omega, \nu, \varphi$, and $f$ as introduced before. There exist a unique $v \in \mathbb{R}$ and a strictly convex function $u \in C^{\infty}(\bar{\Omega})$ solving the boundary value problem

$$
\left\{\begin{aligned}
\operatorname{det} D^{2} u & =e^{v} \cdot f(x, D u) & & \text { in } \Omega, \\
D_{\nu} u & =\varphi & & \text { on } \partial \Omega,
\end{aligned}\right.
$$

provided that there exists a smooth strictly convex function $u_{0}$ satisfying the boundary condition $D_{\nu} u_{0}=\varphi$. The function $u$ is unique up to a constant.

We remark that translating solutions to (1.1) can be viewed as solutions to (1.2), where $v$ denotes the speed.

A situation similar to Theorem 1.1 is considered for the mean curvature flow in [2]. Hypersurfaces of prescribed Gauß curvature subject to Neumann boundary conditions are found in the pioneering paper [4]. The extension to the oblique boundary value problem is made in [7]. Flows of Monge-Ampère type for the Neumann and the second boundary value problem are studied in [6]. In our setting, the situation is more degenerate as neither $f$ nor $\varphi$ do depend on $u$. Thus, both $f$ and $\varphi$ fail to satisfy the crucial monotonicity requirement with respect to $u$. For the second boundary value problem, translating solutions to flows of Gauß curvature type are considered in [5].

As mentioned in [5], methods of [6] can be adapted to the non-parametric logarithmic Gauß curvature flow subject to the second boundary condition. For the Neumann boundary value problem, however, the lack of monotonicity requires a new proof to uniformly bound the oscillation of a solution. Therefore, we establish a generalization of the spatial $C^{1}$-estimates of [4]. Then, we use the translating solutions provided by Theorem 1.2, in particular its uniquely determined speed, to construct an auxiliary barrier function and to obtain uniform spatial $C^{2}$-estimates. Hence, the results of Krylov, Safonov, Evans and Schauder imply uniform bounds on higher derivatives 
for all times, uniformly bounded away from 0 . This allows to show smooth convergence to a translating solution.

The paper is organized as follows: As explained in [6], standard linear parabolic theory and the implicit function theorem imply short-time existence. We show uniform first-order estimates in Sections 2 and 3. Section 4 contains the proof of Theorem 1.2. Having the unique velocity of a translating solution, we can prove uniform a priori $C^{2}$-estimates in Section 5. Finally, in Section 6, we prove smooth convergence to a translating solution. In Appendix A we apply Theorem 1.2 to construct entire graphs of prescribed Gauß curvature. To illustrate the convergence of the flow, we carry out a numerical integration on a planar domain in Appendix B.

\section{2. $\frac{\partial}{\partial t} u$-Estimate.}

Notation 2.1. We write a dot to denote the time derivative, whereas we use indices for the spatial partial derivatives. Let $f_{p_{i}}$ denote a derivative of $f$ with respect to the gradient. For a vector $\xi$ we define $u_{\xi} \equiv \xi^{i} u_{i}$. For the logarithm of $f$ we use $\hat{f} \equiv \log f$. We use the Einstein summation convention and sum over repeated upper and lower indices. The inverse of the Hessian of $u$ is denoted by $\left(u^{i j}\right)=\left(u_{i j}\right)^{-1}$. We remark that - besides in the case $u^{i j}$ - indices are lifted with respect to the Euclidean metric. The letter $c$ denotes a generic positive constant. Furthermore, we may assume that $0 \in \Omega$.

Lemma 2.2. Under the assumptions of Theorem 1.1, there holds

$$
|\dot{u}| \leq \max _{t=0}|\dot{u}|,
$$

as long as a smooth convex solution of (1.1) exists.

Proof. Similar to [6], we consider

$$
r:=(\dot{u})^{2} .
$$

We get the evolution equation

$$
\dot{r}=u^{i j} r_{i j}-2 u^{i j} \dot{u}_{i} \dot{u}_{j}-\hat{f}_{p_{i}} r_{i} .
$$

Hence, $\dot{r} \leq 0$ at a maximum of $r$ in $\Omega \times[0, t]$. Now, assume that a maximum of $r$ on $\bar{\Omega} \times[0, t]$ occurs at $\left(x_{0}, t_{0}\right)$ with $x_{0} \in \partial \Omega$. If $t_{0}=0$ the lemma holds. Thus, in the following, we may assume $t_{0}>0$. If $r$ is constant, then $u$ is a translating solution, and our lemma holds. Otherwise, we get $r_{\beta}\left(x_{0}\right)<0$ from the Hopf boundary point lemma. At $x_{0}$ we compute

$$
0>r_{\beta}=\left((\dot{u})^{2}\right)_{\beta}=2 \dot{u} \dot{u}_{\beta}=2 \dot{u} \frac{\partial}{\partial t} \varphi(x)=0 .
$$

Contradiction. Note, that the assumption $t_{0}>0$ guarantees that $u$ is smooth near $\left(x_{0}, t_{0}\right)$ allowing to interchange differentiation with respect to time and space. 
Integrating the last estimate yields:

Corollary 2.3. As long as a smooth convex solution of (1.1) exists, we obtain the estimate

$$
|u(x, t)| \leq \sup _{\Omega}\left|u_{0}\right|+\sup _{\Omega}|\dot{u}(\cdot, 0)| \cdot t .
$$

\section{Ice-cream cone estimate.}

The following theorem generalizes the $C^{1}$-estimates of [4]. It is essential for our situation, because the oscillation but not the $C^{0}$-norm of the solution is expected to be bounded uniformly in time.

We wish to mention oscillation estimates of Urbas $[\mathbf{9}, 8]$. There, the convexity of $u$ and appropriate growth of $f(x, p)$ in $p$ is used, whereas Theorem 3.1 combines convexity and the boundary condition.

Theorem 3.1 (Ice-cream cone estimate). Let $\Omega \subset \mathbb{R}^{n}$ be a smooth bounded domain, $u: \bar{\Omega} \rightarrow \mathbb{R}$ a smooth strictly convex function with $\left|u_{\beta}\right|$ uniformly bounded on $\partial \Omega$, where $\beta$ is a unit vector field on $\partial \Omega$ such that $\langle\beta, \nu\rangle \geq \widetilde{c}_{\beta}$ for a positive constant $\widetilde{c}_{\beta}>0$ (recall that $\nu$ is the inner unit normal to $\partial \Omega$ ). Then there is a uniform bound for $\sup |D u|$, independent of sup $|u|$.

In view of Lemma 2.2, the result above yields an estimate for the full $C^{1}$ norm of solutions $u$ to (1.1). Note, that only the estimate for the derivatives of $u$ is uniform in time.

Proof. In the name of the theorem we want to emphasize that our proof uses balls and cones, similar to ice-cream placed in a cone of waffle. We argue by contradiction. Assume that there exists a point $x_{0}$, where $|D u|$ is maximal and equal to $M$. If $M$ is larger than a suitably chosen constant $M_{0}$ we will find a contradiction. As $u$ is strictly convex, we see that $x_{0} \in \partial \Omega$. At $x_{0}$ we find a tangential direction $\xi_{0}$ such that $\left\langle D u\left(x_{0}\right), \xi_{0}\right\rangle$ is maximal compared to all other tangential directions. Here and later, unit vectors are called directions. We wish to prove a lower estimate for $\left\langle D u\left(x_{0}\right), \xi_{0}\right\rangle$ in terms of $M$.

Let $\xi_{1}$ be a direction such that $\left\langle D u\left(x_{0}\right), \xi_{1}\right\rangle=M$. Similar to [7], we decompose a direction $\xi$ using $\beta$ and a tangential vector $\tau(\xi)$ as

$$
\xi=\tau(\xi)+\frac{\langle\nu, \xi\rangle}{\langle\beta, \nu\rangle} \beta,
$$

where

$$
\tau(\xi)=\xi-\langle\nu, \xi\rangle \nu-\frac{\langle\nu, \xi\rangle}{\langle\beta, \nu\rangle} \beta^{T}, \quad \beta^{T}=\beta-\langle\beta, \nu\rangle \nu .
$$


Note, that $|\tau(\xi)|$ is bounded by assumption. Decomposing $\xi_{1}$, we get

$$
\begin{aligned}
M & =\left\langle D u, \xi_{1}\right\rangle=\left\langle D u, \tau\left(\xi_{1}\right)\right\rangle+\frac{\left\langle\nu, \xi_{1}\right\rangle}{\langle\beta, \nu\rangle}\langle D u, \beta\rangle \\
& \leq\left|\tau\left(\xi_{1}\right)\right| \cdot \max _{\substack{\tau \in T_{x_{0}} \partial \Omega \\
|\tau|=1}}\langle D u, \tau\rangle+c \\
& =\left|\tau\left(\xi_{1}\right)\right| \cdot\left\langle D u\left(x_{0}\right), \xi_{0}\right\rangle+c .
\end{aligned}
$$

Hence, we deduce that $\left\langle D u\left(x_{0}\right), \xi_{0}\right\rangle \geq \frac{M}{c}$, as long as $M \geq M_{0}$ and $M_{0}$ is chosen sufficiently large. For a direction $\xi$ near $\xi_{0}$, say $\left|\xi-\xi_{0}\right|<\varepsilon=\frac{1}{2 c}<1$, we obtain

$$
\left\langle D u\left(x_{0}\right), \xi\right\rangle=\left\langle D u, \xi_{0}\right\rangle+\left\langle D u, \xi-\xi_{0}\right\rangle \geq \frac{M}{c}-M\left|\xi-\xi_{0}\right| \geq \varepsilon M .
$$

From the convexity of $u$, we deduce that $\langle D u(y), \xi\rangle \geq \varepsilon M$ for all points $y \in \bar{\Omega}$ of the form $y=x_{0}+\lambda \cdot \xi$. Here, $\lambda>0$ and $\xi$ are chosen such that $\left|\xi-\xi_{0}\right| \leq \varepsilon$ and $x_{0}+t \cdot \lambda \cdot \xi \in \bar{\Omega}$ for all $t \in[0,1]$.

The uniform boundedness of the principal curvatures of $\partial \Omega \subset \mathbb{R}^{n}$ implies that there exist $R>0$ and $x_{1} \in \partial \Omega$ such that $\left|x_{0}-x_{1}\right|>2 R$, and, especially, any $x \in B_{R}\left(x_{1}\right) \cap \partial \Omega$ can be written in the form $x_{0}+\lambda \cdot \xi$, as described above. Thus, according to (3.2), $|D u| \geq \varepsilon M$ in $\partial \Omega \cap B_{R}\left(x_{1}\right)$. Due to our construction, we have

$$
\inf _{x \in B_{R}\left(x_{1}\right) \cap \partial \Omega} u(x)>u\left(x_{0}\right) .
$$

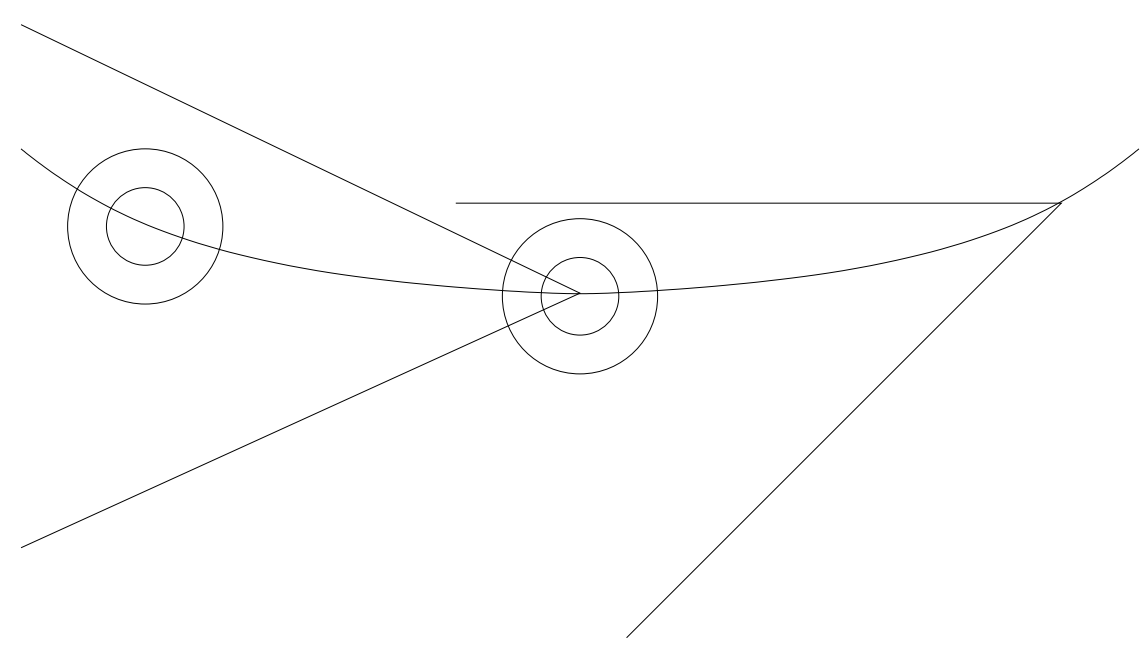

Figure 1. Ice-cream cone estimate.

Figure 1 shows a part of $\partial \Omega$ and two cones corresponding to the directions $\xi$ as well as two pairs of concentric balls. The larger ones are the balls $B_{R}$ mentioned above, the smaller ones are introduced in the following. 
Now, we proceed iteratively. Note, that $R$ and $\varepsilon$ can be chosen as fixed constants, independent of the point $x_{0}$. As long as $\left|D u\left(x_{i}\right)\right| \geq M \varepsilon^{i} \geq M_{0}$, we can find a further point $x_{i+1}$ as we went from $x_{0}$ to $x_{1}$. Thus, for $M=$ $\sup |D u|$ sufficiently large, we can construct a sequence of points $\left\{x_{i}\right\}_{i=0, \ldots, N}$ of arbitrarily large length $N$, satisfying for all $i \geq 1$

$$
|D u| \geq M \varepsilon^{i} \quad \text { on } \partial \Omega \cap B_{R}\left(x_{i}\right)
$$

and

$$
\inf _{x \in B_{R}\left(x_{i}\right) \cap \partial \Omega} u(x)>u\left(x_{i-1}\right) .
$$

Since $\partial \Omega$ has finite measure and bounded principal curvatures, there is an upper bound $N_{0}(\rho)$ on the number of pairwise disjoint restricted balls $B_{\rho}\left(y_{j}\right) \cap \partial \Omega$ for fixed $\rho>0$ and $y_{j} \in \partial \Omega$.

Hence, if $M=\sup |D u|>M_{0} \varepsilon^{-N_{0}\left(\frac{R}{2}\right)}$, there will be two points $x_{i_{0}}, x_{j_{0}}$ with $i_{0}>j_{0}>0$ such that

$$
B_{\frac{R}{2}}\left(x_{i_{0}}\right) \cap B_{\frac{R}{2}}\left(x_{j_{0}}\right) \cap \partial \Omega \neq \emptyset .
$$

But $x_{j_{0}} \in B_{R}\left(x_{i_{0}}\right)$ implies

$$
u\left(x_{j_{0}}\right)<u\left(x_{j_{0}+1}\right)<\cdots<u\left(x_{i_{0}-1}\right)<\inf _{x \in B_{R}\left(x_{i_{0}}\right) \cap \partial \Omega} u(x) \leq u\left(x_{j_{0}}\right) .
$$

Contradiction.

\section{Existence of translating solutions.}

This section is devoted to the proof of Theorem 1.2. That is, we construct solutions to the elliptic problem

$$
\left\{\begin{aligned}
v & =\log \operatorname{det} D^{2} u-\log f(x, D u) & & \text { in } \Omega, \\
u_{\beta} & =\varphi & & \text { on } \partial \Omega .
\end{aligned}\right.
$$

The absence of any monotonicity property in $u$, both in $f$ as well as in the boundary condition $\varphi$, limits seriously the existence of solutions.

Step 1. Here, we show that for given $\varepsilon>0$ and $v \in \mathbb{R}$ there is a unique solution $u_{\varepsilon, v}$ of

$$
\left(*_{\varepsilon, v}\right) \quad\left\{\begin{aligned}
\operatorname{det} D^{2} u & =e^{v} f(x, D u) e^{\varepsilon u} & & \text { in } \Omega, \\
u_{\beta} & =\varphi & & \text { on } \partial \Omega .
\end{aligned}\right.
$$

Note, that the dependence on $v$ is continuous and strictly decreasing. In fact, we have the explicit relation

$$
u_{\varepsilon, v}=u_{\varepsilon, 0}-\frac{v}{\varepsilon}
$$

To show the unique existence of $u_{\varepsilon, v}$, we will derive an a priori $C^{0}$-bound, then the ice-cream cone estimate, Theorem 3.1, yields the $C^{1}$-bound. Having controlled the full $C^{1}$-norm, we can estimate the $C^{2}$-norm exactly as in Urbas [7], since the strict monotonicity assumption on $\varphi$ is not used for this 
part. A detailed argument, applied to the parabolic case, is given in the next section. Bounds for higher $C^{k}$-norms follow via the estimates due to Krylov, Safonov, Evans, and from Schauder theory.

To get the $C^{0}$-bound, we define suitable barriers for $\left(*_{\varepsilon, 0}\right)$. Recall, that $u_{0}$ is a convex function satisfying $\left(u_{0}\right)_{\beta}=\varphi$ on $\partial \Omega$. Define $u_{\varepsilon}^{ \pm}=u_{0} \pm M / \varepsilon$, where $M>0$ will be chosen later. Then

$$
\frac{\operatorname{det} D^{2} u_{\varepsilon}^{ \pm}}{f\left(x, D u_{\varepsilon}^{ \pm}\right) e^{\varepsilon u_{\varepsilon}^{ \pm}}}=\frac{\operatorname{det} D^{2} u_{0}}{f\left(x, D u_{0}\right) e^{\varepsilon u_{0} \pm M}}=g(x) e^{-\varepsilon u_{0}} e^{\mp M},
$$

with $c^{-1}<g(x)<c$. Hence, restricting ourselves to $\varepsilon<1$, there exists a large constant $M>0$, not depending on $\varepsilon$, such that $u_{\varepsilon}^{+}$is a strict supersolution and $u_{\varepsilon}^{-}$is a strict subsolution of $\left(*_{\varepsilon, 0}\right)$. This implies, that

$$
u_{\varepsilon}^{-}<u_{\varepsilon, 0}<u_{\varepsilon}^{+},
$$

or equivalently,

$$
\left|u_{\varepsilon, v}-\left(u_{0}-\frac{v}{\varepsilon}\right)\right|<\frac{M}{\varepsilon} .
$$

Step 2. Now, we consider the limit $\varepsilon \rightarrow 0$. In general, we cannot expect that the sup bounds for $u_{\varepsilon, v}$ can be obtained uniformly in $\varepsilon$. In fact, it follows from the maximum principle that only for a unique $v$, there is a solution to $\left(*_{\varepsilon, v}\right)$ with $\varepsilon=0$. Observe, that (4.2) implies that

$$
u_{\varepsilon,+M}<u_{0}<u_{\varepsilon,-M} .
$$

Therefore, for every $\varepsilon>0$ we can find a unique $v_{\varepsilon} \in(-M, M)$ such that $u_{\varepsilon, v_{\varepsilon}}(0)=u_{0}(0)$. Note, that (4.2) does not suffice to control the oscillation of $u_{\varepsilon, v_{\varepsilon}}$, uniformly in $\varepsilon$. We employ the ice-cream cone estimate to bound $u_{\varepsilon, v_{\varepsilon}}$ uniformly in $C^{1}$. Again, uniform $C^{1}$-bounds imply uniform higher $C^{k}$-bounds.

Now, we choose a sequence $\varepsilon_{i} \rightarrow 0$ as $i \rightarrow \infty$. Since $v_{\varepsilon_{i}}$ is bounded, there exists a subsequence, relabeled, such that $v_{\varepsilon_{i}} \rightarrow v^{\infty}$ and $u_{\varepsilon_{i}, v_{\varepsilon_{i}}} \rightarrow u_{\mathrm{ell}}^{\infty}$ in any $C^{k}$-norm. This completes the proof of Theorem 1.2.

The extension $u^{\infty}(x, t):=u_{\mathrm{ell}}^{\infty}(x)+v^{\infty} t$ is a translating solution, as, by construction, $u^{\infty}$ satisfies

$$
\left\{\begin{aligned}
v^{\infty}=\dot{u}^{\infty} & =\log \operatorname{det} D^{2} u^{\infty}-\log f\left(x, D u^{\infty}\right) & & \text { in } \Omega, \\
u_{\beta}^{\infty} & =\varphi & & \text { on } \partial \Omega .
\end{aligned}\right.
$$

\section{Parabolic $C^{2}$-estimates.}

The following argument is a modification of the proofs in $[4,6]$ and $[7]$. We use the translating solution $u^{\infty}$, especially, its speed $v^{\infty}$, to construct an auxiliary barrier function.

Assume that $u_{\text {ell }}^{\infty}>u_{0}$. We define

$$
\widetilde{\varphi}(x, z)=\varphi(x)+\left(z-u_{\mathrm{ell}}^{\infty}\right) .
$$


Due to uniform estimates on the gradient of $u$, we can find a positive constant $\mu_{0}$ such that

$$
\min \left\{f(x, D u), f\left(x, D u_{\text {ell }}^{\infty}\right)\right\} \geq \mu_{0} .
$$

For $0<\rho<1$, consider the elliptic boundary value problem

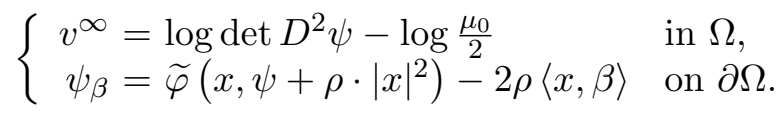

We wish to show a uniform a priori $C^{2}$-estimate for $\psi$. Theorem 3.1 gives an estimate for the gradient. Similarly to [7], bounds on the second derivatives follow. It is here that the smallness of $\|\nu-\beta\|_{C^{1}}$ is used. Thus, it remains to prove uniform $C^{0}$-estimates. Note, that a convex solution $\psi$ cannot satisfy $\psi_{\beta}(x)>0$ for all $x \in \partial \Omega$. Hence, the upper bound on $\psi$ follows since $\widetilde{\varphi}(x, z) \rightarrow \infty$ uniformly as $z \rightarrow \infty$. For the lower bound, we consider $\psi-u_{\mathrm{ell}}^{\infty}$. Applying the maximum principle to the differential inequality

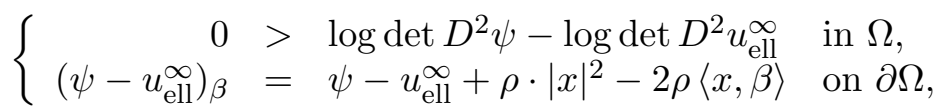

we see that $\psi-u^{\infty}$ cannot attain an interior minimum. If a minimum occurs on $\partial \Omega$, we get

$$
0 \leq\left(\psi-u_{\mathrm{ell}}^{\infty}\right)_{\beta}=\psi-u_{\mathrm{ell}}^{\infty}+\rho \cdot|x|^{2}-2 \rho\langle x, \beta\rangle .
$$

Thus, $\psi$ is uniformly bounded below, a solution to (5.1) exists. Due to the uniform $C^{2}$-estimates, we can fix $\lambda>0$ such that

$$
\left(\psi_{i j}\right) \geq \lambda \mathrm{Id}
$$

Furthermore, these estimates allow to fix $\rho>0$ such that $\bar{\psi}:=\psi+\rho \cdot|x|^{2}$ satisfies

$$
\begin{cases}v^{\infty}>\log \operatorname{det} D^{2} \bar{\psi}-\log \mu_{0} & \text { in } \Omega \\ \bar{\psi}_{\beta}=\widetilde{\varphi}(x, \bar{\psi}) & \text { on } \partial \Omega .\end{cases}
$$

Applying the maximum principle, we get $u_{\text {ell }}^{\infty} \leq \bar{\psi}$. We extend $\psi$ and $\bar{\psi}$ by setting $\psi(x, t):=\psi(x)+t \cdot v^{\infty}, \bar{\psi}(x, t):=\bar{\psi}(x)+t \cdot v^{\infty}$, respectively. Thus, $u \leq u^{\infty} \leq \bar{\psi}$, where $u^{\infty}$ is the translating solution defined in Section 4. We get for $x \in \partial \Omega$

$$
\left(\bar{\psi}_{\beta}-u_{\beta}\right)(x, t)=\bar{\psi}_{\beta}(x, 0)-\varphi(x)=\left(\bar{\psi}-u^{\infty}\right)(x, 0)=\left(\bar{\psi}-u^{\infty}\right)(x) \geq 0 .
$$

Furthermore, for a sufficiently small $\delta_{0}>0$,

$$
(\psi-u)_{\beta}=\left(\bar{\psi}-\rho \cdot|x|^{2}-u\right)_{\beta} \geq-2 \rho\langle x, \beta\rangle \geq \delta_{0}>0 \quad \text { on } \partial \Omega,
$$

provided that $\beta$ is $C^{0}$-close to $\nu$. Here, we used that $0 \in \Omega$ implying $\langle x, \nu\rangle<$ 0 . Using these preparations, we prove a priori $C^{2}$-estimates similarly to $[6]$ and [7]. For the reader's convenience, we repeat the arguments incorporating the necessary modifications to the parabolic case. 
5.1. Preliminary results. Assume, that a smooth solution $u$ of our flow equation (1.1) exists on the time interval $[0, T]$. We will use the letter $\tau$ to indicate a direction tangential to $\partial \Omega$.

Lemma 5.1 (Mixed $C^{2}$-estimates at the boundary). Let $u$ be a solution of (1.1). Then $\left|u_{\tau \beta}\right|$ remains uniformly bounded on $\partial \Omega$.

Proof. We represent $\partial \Omega$ locally as graph $\omega$ over its tangent plane at a fixed point $x_{0} \in \partial \Omega$ such that locally $\Omega=\left\{\left(\hat{x}, x^{n}\right): x^{n}>\omega(\hat{x})\right\}$. Let us extend $\beta$ and $\varphi$ smoothly. At $x_{0}$, differentiating the oblique boundary condition

$$
\beta^{i}(\hat{x}) u_{i}(\hat{x}, \omega(\hat{x}))=\varphi(\hat{x}, \omega(\hat{x})), \quad \hat{x} \in \mathbb{R}^{n-1},
$$

with respect to tangential directions $\hat{x}^{j}, 1 \leq j \leq n-1$,

$$
\beta_{j}^{i} u_{i}+\beta^{i} u_{i j}+\beta^{i} u_{i n} \omega_{j}=\varphi_{j}+\varphi_{n} \omega_{j},
$$

we obtain at $x_{0} \equiv\left(\hat{x}_{0}, \omega\left(\hat{x}_{0}\right)\right) \in \partial \Omega$ a bound for $\beta^{i} u_{i j}$. Thereby, we use the gradient estimate for $u$ and $D \omega\left(\hat{x}_{0}\right)=0$. Multiplying with $\tau^{j}$ gives the result.

Lemma 5.2 (Double oblique $C^{2}$-estimates at the boundary). For any solution of (1.1), $\left|u_{\beta \beta}\right|$ is uniformly bounded on $\partial \Omega$.

Proof. Note that $u_{\beta \beta}>0$ as $u(\cdot, t)$ is strictly convex for each $t$. We keep the geometric setting of the proof of Lemma 5.1 with $x_{0} \in \partial \Omega$. From (1.1) we obtain

$$
\dot{u}_{k}=u^{i j} u_{i j k}-\left(\hat{f}_{k}+\hat{f}_{p_{i}} u_{i k}\right)
$$

We define

$$
L w:=\dot{w}-u^{i j} w_{i j}+\hat{f}_{p_{i}} w_{i} .
$$

We can find appropriate extensions of $\beta$ and $\varphi$ such that

$$
\left|L\left(\beta^{k} u_{k}-\varphi(x)\right)\right| \leq c \cdot\left(1+\operatorname{tr}\left(u^{i j}\right)\right) .
$$

We choose $\delta>0$ sufficiently small and define $\Omega_{\delta}:=\Omega \cap B_{\delta}\left(x_{0}\right)$. Set

$$
\vartheta:=d-\mu d^{2},
$$

where $\mu \gg 1$ is chosen sufficiently large, and $d$ denotes the distance to $\partial \Omega$. We will show that in $\Omega_{\delta}$ there holds $L \vartheta \geq \frac{\varepsilon}{3} \operatorname{tr}\left(u^{i j}\right)$ for a small constant $\varepsilon>0$, depending only on a positive lower bound for the principal curvatures of $\partial \Omega$. Next,

$$
\begin{aligned}
L \vartheta & =-u^{i j} d_{i j}+2 \mu u^{i j} d_{i} d_{j}+2 \mu u^{i j} d d_{i j}+\hat{f}_{p_{i}}\left(d_{i}-2 \mu d d_{i}\right) \\
& \geq-u^{i j} d_{i j}+2 \mu u^{i j} d_{i} d_{j}-c \mu d\left(1+\operatorname{tr}\left(u^{i j}\right)\right)-c .
\end{aligned}
$$


We use the strict convexity of $\partial \Omega,\left|D d-e_{n}\right| \leq c \delta,\left|u^{k l}\right| \leq \operatorname{tr}\left(u^{i j}\right), 1 \leq k, l \leq$ $n$, and the inequality for arithmetic and geometric means

$$
\begin{aligned}
L \vartheta \geq & \varepsilon \operatorname{tr}\left(u^{i j}\right)+\mu u^{n n}-c \mu \delta\left(1+\operatorname{tr}\left(u^{i j}\right)\right)-c \\
\geq & \frac{n}{3}\left(\operatorname{det}\left(u^{i j}\right)\right)^{\frac{1}{n}} \cdot \varepsilon^{\frac{n-1}{n}} \cdot \mu^{\frac{1}{n}}+\frac{2}{3} \varepsilon \operatorname{tr}\left(u^{i j}\right) \\
& -c \mu \delta\left(1+\operatorname{tr}\left(u^{i j}\right)\right)-c .
\end{aligned}
$$

More precisely, we used

$$
\frac{\varepsilon \operatorname{tr}\left(u^{i j}\right)+\mu u^{n n}}{3} \geq \frac{n}{3} \varepsilon^{\frac{n-1}{n}} \mu^{\frac{1}{n}}\left(\prod_{i=1}^{n} u^{i i}\right)^{\frac{1}{n}},
$$

and, assuming $\left(u^{i j}\right)_{i, j<n}$ is diagonal,

$$
\begin{aligned}
\operatorname{det}\left(u^{i j}\right) & =\operatorname{det}\left(\begin{array}{ccccc}
u^{11} & 0 & \cdots & 0 & u^{1 n} \\
0 & \ddots & \ddots & \vdots & \vdots \\
\vdots & \ddots & \ddots & 0 & \vdots \\
0 & \cdots & 0 & u^{n-1 n-1} & u^{n-1 n} \\
u^{1 n} & \cdots & \cdots & u^{n-1 n} & u^{n n}
\end{array}\right) \\
& =\prod_{i=1}^{n} u^{i i}-\sum_{i<n}\left|u^{n i}\right|^{2} \prod_{\substack{j \neq i \\
j<n}} u^{j j} \leq \prod_{i=1}^{n} u^{i i} .
\end{aligned}
$$

Since

$$
\operatorname{det}\left(u^{i j}\right)=\frac{1}{\operatorname{det}\left(u_{i j}\right)}=\exp (-\hat{f}-\dot{u})
$$

$\operatorname{det}\left(u^{i j}\right)$ is uniformly bounded from below by a positive constant. We may choose $\mu$ so large, that

$$
\frac{n}{3}\left(\operatorname{det}\left(u^{i j}\right)\right)^{\frac{1}{n}} \cdot \varepsilon^{\frac{n-1}{n}} \cdot \mu^{\frac{1}{n}} \geq c+1 .
$$

For $\delta \leq \frac{1}{c \mu} \min \left\{1, \frac{1}{3} \varepsilon\right\}$, we get

$$
L \vartheta \geq \frac{1}{3} \varepsilon \operatorname{tr}\left(u^{i j}\right) .
$$

Furthermore, $\vartheta \geq 0$ on $\partial \Omega_{\delta}$, if $\delta$ is chosen smaller if necessary.

Let $l$ be an affine linear function such that $l\left(x_{0}\right)=0$ and

$$
l \geq \beta^{i}\left(u_{0}\right)_{i}-\varphi \text { in } \Omega_{\delta} .
$$

For constants $A, B>0$, consider the function

$$
\Theta:=A \vartheta+B\left|x-x_{0}\right|^{2}-\left(\beta^{i} u_{i}-\varphi(x)\right)+l .
$$

We fix $B \gg 1$, get $\Theta \geq 0$ on $\partial \Omega_{\delta}$, and deduce for $A \gg B$ that $L \Theta \geq 0$, since $\operatorname{tr}\left(u^{i j}\right)$ is bounded from below by a positive constant. The maximum principle yields $\Theta \geq 0$ in $\Omega_{\delta}$. As $\Theta\left(x_{0}\right)=0$, we conclude that $\Theta_{\beta}\left(x_{0}\right) \geq 0$ implying $u_{\beta \beta} \leq c$. 
Lemma 5.3. For a solution of (1.1), there holds

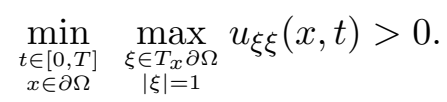

Proof. We have already seen that there is a uniform positive lower bound for $\operatorname{det} D^{2} u$. Again, at a fixed boundary point, we may choose a coordinate system, such that $e_{n}$ is equal to the inner unit normal of $\partial \Omega$ and $\left(u_{i j}\right)_{i, j<n}$ is diagonal. Similarly to (5.4), we estimate

$$
\operatorname{det}\left(u_{i j}\right)=\prod_{i=1}^{n} u_{i i}-\sum_{i<n}\left|u_{n i}\right|^{2} \prod_{\substack{j \neq i \\ j<n}} u_{j j} \leq \prod_{i=1}^{n} u_{i i}
$$

We decompose $\nu$ as

$$
\nu=\frac{1}{\langle\beta, \nu\rangle}\left(\beta-\beta^{T}\right),
$$

$\beta^{T}$ as in the proof of Theorem 3.1, and get in view of Lemmata 5.1 and 5.2

$$
u_{\nu \nu} \leq \frac{1}{\langle\beta, \nu\rangle^{2}}\left(\left|\beta^{T}\right|^{2} \cdot \max _{i<n} u_{i i}+c\right) \text {. }
$$

Finally, the claimed bound follows from (5.5).

5.2. Remaining $\boldsymbol{C}^{\mathbf{2}}$-estimates. Similarly to [7], we define

$$
w(x, \xi, t):=e^{\alpha(\psi-u)+\gamma \cdot|D u|^{2}} \cdot u_{\xi \xi}
$$

for $(x, \xi, t) \in \bar{\Omega} \times S^{n-1} \times[0, T]$ and positive constants $\alpha, \gamma$ to be fixed later. We assume that $w$, restricted to boundary points and tangential directions, attains its maximum at $x_{w} \in \partial \Omega$ in a tangential direction which we may take to be $e_{1}$, and $t_{w} \in[0, T]$. We may assume that $t_{w}>0$. Furthermore, we fix Euclidean coordinates such that $e_{n}$ is the inner normal direction and $\left(u_{i j}\right)_{i, j<n}\left(x_{w}\right)$ is diagonal. Decompose $e_{1}$ as

$$
e_{1}=\tau\left(e_{1}\right)+\frac{\left\langle\nu, e_{1}\right\rangle}{\langle\beta, \nu\rangle} \beta
$$

where

$$
\tau\left(e_{1}\right)=\tau=e_{1}-\left\langle\nu, e_{1}\right\rangle \nu-\frac{\left\langle\nu, e_{1}\right\rangle}{\langle\beta, \nu\rangle} \beta^{T}, \quad \beta^{T}=\beta-\langle\beta, \nu\rangle \nu .
$$

Note that $\tau$ is tangential, but not necessarily of unit length. For smoothly extended $\beta$ and $\varphi$, we differentiate the boundary condition and obtain on $\partial \Omega$

$$
\frac{2\left\langle\nu, e_{1}\right\rangle}{\langle\nu, \beta\rangle} u_{\beta \tau}=\frac{2\left\langle\nu, e_{1}\right\rangle}{\langle\nu, \beta\rangle}\left(\varphi_{j} \tau^{j}-\tau^{j} \beta_{j}^{i} u_{i}\right)=: \chi(x, D u) .
$$

On the boundary, we get

$$
u_{11}=u_{\tau \tau}+\chi+\frac{\left\langle\nu, e_{1}\right\rangle^{2}}{\langle\beta, \nu\rangle^{2}} u_{\beta \beta}
$$


Since $\chi\left(x_{w}, \cdot\right)=0$, the function

$$
\widetilde{w}(x, t):=e^{\alpha(\psi-u)+\gamma|D u|^{2}}\left(u_{11}-\chi\right)
$$

satisfies $\widetilde{w}\left(x_{w}, t_{w}\right)=w\left(x_{w}, \tau, t_{w}\right)$, moreover, for all $x \in \partial \Omega$ and $t \in[0, T]$ (5.7) $\widetilde{w}(x, t)$

$$
\begin{aligned}
= & e^{\alpha(\psi-u)+\gamma|D u|^{2}}(x, t)\left\{u_{\tau \tau}(x, t)+\frac{\left\langle\nu, e_{1}\right\rangle^{2}}{\langle\beta, \nu\rangle^{2}} u_{\beta \beta}(x, t)\right\} \\
\leq & \left\{1-\left\langle\nu, e_{1}\right\rangle^{2}\left(1-\frac{\left|\beta^{T}\right|^{2}}{\langle\beta, \nu\rangle^{2}}\right)-\frac{2\left\langle\nu, e_{1}\right\rangle\left\langle\beta^{T}, e_{1}\right\rangle}{\langle\beta, \nu\rangle}\right\} \widetilde{w}\left(x_{w}, t_{w}\right) \\
& +c\left\langle\nu, e_{1}\right\rangle^{2} e^{\alpha(\psi-u)+\gamma|D u|^{2}}(x, t) \\
\leq & \left\{1+c\left\langle\nu, e_{1}\right\rangle^{2}-\frac{2\left\langle\nu, e_{1}\right\rangle\left\langle\beta^{T}, e_{1}\right\rangle}{\langle\beta, \nu\rangle}+c \frac{\left\langle\nu, e_{1}\right\rangle^{2}}{\max _{\xi \in T_{x} \partial \Omega} u_{\xi \xi}(x)}\right\} \widetilde{w}\left(x_{w}, t_{w}\right) \\
\leq \xi \mid=1 & \left\{1+c_{1}\left\langle\nu, e_{1}\right\rangle^{2}-\frac{2\left\langle\nu, e_{1}\right\rangle\left\langle\beta^{T}, e_{1}\right\rangle}{\langle\beta, \nu\rangle}\right\} \widetilde{w}\left(x_{w}, t_{w}\right) .
\end{aligned}
$$

Now, Lemma 5.3 ensures that we can choose $c_{1}$ in the last inequality independent of $\alpha$ and $\gamma$.

We may assume that $c_{1}$ in (5.7) is chosen sufficiently large and $\beta$ is sufficiently close to $\nu$ such that the expression in the last curly brackets in (5.7) is bounded below by $\frac{1}{2}$.

We define on $\bar{\Omega} \times[0, T]$

$$
W:=\frac{e^{\alpha(\psi-u)+\gamma|D u|^{2}}\left(u_{11}-\chi\right)}{1+c_{1}\left\langle\nu, e_{1}\right\rangle^{2}-\frac{2\left\langle\nu, e_{1}\right\rangle\left\langle\beta^{T}, e_{1}\right\rangle}{\langle\beta, \nu\rangle}} .
$$

Assume that $W$ attains its maximum at $\left(x_{W}, t_{W}\right)$ and $t_{W}>0$.

First, we address the case $x_{W} \in \partial \Omega$. Observe that $W\left(x_{W}, t_{W}\right) \leq \widetilde{w}\left(x_{w}, t_{w}\right)$ $=W\left(x_{w}, t_{w}\right)$. At $\left(x_{w}, t_{w}\right)$, we get $W_{\beta} \leq 0$, which implies that

$$
u_{11 \beta}+\alpha \delta_{0} u_{11} \leq c\left(1+(1+\gamma) u_{11}\right),
$$

using $\delta_{0}$ from (5.3). At $x_{w}$, keeping the notation of Lemma 5.1, we differentiate the boundary condition $u_{\beta}=\varphi$ twice in direction $e_{1}$. The a priori estimates obtained so far, and the fact that $D^{2} u$, restricted to tangential directions, is diagonal, yield

$$
u_{\beta 11} \geq-c-2 \beta_{1}^{1} u_{11}-2 \beta_{1}^{n} u_{n 1} .
$$

Then, combining (5.8) and (5.9) implies

$$
c\left(1+(1+\gamma) u_{11}\right) \geq \alpha \delta_{0} u_{11}-c .
$$


For $\alpha=\alpha(\gamma)$ sufficiently large, we get an upper bound on $u_{11}\left(x_{w}, t_{w}\right)$. This completes the $C^{2}$-estimates, if $W$ attains its maximum on $\partial \Omega$.

Now, we consider the case that $W$ attains its maximum at $\left(x_{W}, t_{W}\right)$, $x_{W} \in \Omega$. We use

$$
\Gamma=-\log \left(1+c_{1}\left\langle\nu, e_{1}\right\rangle^{2}-\frac{2\left\langle\nu, e_{1}\right\rangle\left\langle\beta^{T}, e_{1}\right\rangle}{\langle\beta, \nu\rangle}\right)
$$

in the following calculations. $\Gamma$ is well-defined as the argument of the logarithm is bounded below by a positive constant. Moreover, the $C^{2}(\bar{\Omega})$-norm of $\Gamma$ is uniformly bounded independent of $\alpha$ and $\gamma$. We use that

$$
\log W=\alpha \cdot(\psi-u)+\gamma \cdot|D u|^{2}+\log \left(u_{11}-\chi\right)+\Gamma
$$

attains its maximum at $x_{W}$. Of course, we may assume that $1 \leq\left(u_{11}-\right.$ $\chi)\left(x_{W}, t_{W}\right)$. At $\left(x_{W}, t_{W}\right)$, we get

$$
\begin{aligned}
& 0 \leq \frac{\dot{W}}{W}=\alpha(\dot{\psi}-\dot{u})+2 \gamma u^{k} \dot{u}_{k}+\frac{\dot{u}_{11}-\frac{d}{d t} \chi}{u_{11}-\chi}, \\
& 0=\frac{W_{i}}{W}=\alpha(\psi-u)_{i}+2 \gamma u^{k} u_{k i}+\frac{u_{11 i}-D_{i} \chi}{u_{11}-\chi}+\Gamma_{i},
\end{aligned}
$$

and, in the matrix sense,

$$
\begin{aligned}
0 \geq & \frac{W_{i j}}{W}-\frac{W_{i} W_{j}}{W^{2}} \\
= & \alpha(\psi-u)_{i j}+2 \gamma u_{j}^{k} u_{k i}+2 \gamma u^{k} u_{k i j} \\
& +\frac{u_{11 i j}-D_{i j} \chi}{u_{11}-\chi}-\frac{\left(u_{11 i}-D_{i} \chi\right)\left(u_{11 j}-D_{j} \chi\right)}{\left(u_{11}-\chi\right)^{2}}+\Gamma_{i j},
\end{aligned}
$$

where we have used that $\Gamma$ is time-independent. We use $D$. and $\frac{d}{d t}$ to indicate that the chain rule has not yet been applied. In the rest of the section, we drop the argument, if we evaluate at $\left(x_{W}, t_{W}\right)$. We get

$$
0 \geq u^{i j}(\log W)_{i j}-\dot{W} .
$$

Estimates for the time derivatives of $\psi$ and $u$, the strict convexity of $\psi$ (5.2), the fact that $\Gamma \in C^{2}$ with uniform bounds, and the differentiated flow equation (1.1) yield

$$
\begin{aligned}
0 \geq & 2 \gamma \Delta u+\frac{1}{u_{11}-\chi}\left(u^{i r} u^{j s} u_{i j 1} u_{r s 1}\right) \\
& -u^{i j} \frac{\left(u_{11 i}-D_{i} \chi\right)\left(u_{11 j}-D_{j} \chi\right)}{\left(u_{11}-\chi\right)^{2}} \\
& +\frac{1}{u_{11}-\chi}\left(\hat{f}_{p_{i}} u_{i 11}-c-c \cdot\left|D^{2} u\right|^{2}\right)+2 \gamma u^{k} \hat{f}_{p_{i}} u_{i k} \\
& +\frac{1}{u_{11}-\chi}\left(\frac{d}{d t} \chi-u^{i j} D_{i j} \chi\right)-c(\alpha+\gamma)+(\alpha \lambda-c) \operatorname{tr}\left(u^{i j}\right) .
\end{aligned}
$$


Direct calculations and (1.1) imply

$$
\frac{d}{d t} \chi-u^{i j} D_{i j} \chi \geq-c\left(1+\left|D^{2} u\right|+\operatorname{tr}\left(u^{i j}\right)\right) .
$$

We use $\frac{W_{i}}{W}=0$ to get

$$
2 \gamma u^{k} \hat{f}_{p_{i}} u_{i k}+\frac{\hat{f}_{p_{i}} u_{11 i}}{u_{11}-\chi} \geq-c \cdot \alpha-\frac{c \cdot\left(1+\left|D^{2} u\right|\right)}{u_{11}-\chi}-c .
$$

Now, these estimates are applied to (5.10). Let $\vartheta \in\left(0, \frac{1}{2}\right)$ be a small constant, to be fixed later. First, we assume that, still at $\left(x_{W}, t_{W}\right)$,

$$
(1-\vartheta) u_{\eta \eta} \equiv(1-\vartheta) \max _{|\xi|=1} u_{\xi \xi} \leq\left(u_{11}-\chi\right) .
$$

Here, a direction $\eta,|\eta|=1$, is chosen which corresponds to a maximal eigenvalue. Schwarz's inequality gives

$$
u^{i j}\left(u_{11 i}-D_{i} \chi\right)\left(u_{11 j}-D_{j} \chi\right) \leq(1+\vartheta) u^{i j} u_{11 i} u_{11 j}+\frac{c}{\vartheta} u^{i j} D_{i} \chi D_{j} \chi .
$$

From the definition of $\eta$, we get

$$
u^{i r} u^{j s} u_{i j 1} u_{r s 1} \geq \frac{\max _{|\xi|=1} u^{j s} u_{\xi j 1} u_{\xi s 1}}{u_{\eta \eta}} \geq \frac{1-\vartheta}{u_{11}-\chi} u^{i j} u_{11 i} u_{11 j} .
$$

Using $\vartheta \leq \frac{1}{2}$ and $\frac{W_{i}}{W}=0$, we get

$$
\begin{aligned}
& u^{i r} u^{j s} u_{i j 1} u_{r s 1}-u^{i j} \frac{1}{u_{11}-\chi}\left(u_{11 i}-D_{i} \chi\right)\left(u_{11 j}-D_{j} \chi\right) \\
& \geq u^{i r} u^{j s} u_{i j 1} u_{r s 1}-(1+\vartheta) \frac{1}{u_{11}-\chi} u^{i j} u_{11 i} u_{11 j}-\frac{c}{\vartheta(1-\vartheta)} \frac{1}{u_{\eta \eta}} u^{i j} D_{i} \chi D_{j} \chi \\
& \geq-\vartheta \frac{2}{u_{11}-\chi} u^{i j} u_{11 i} u_{11 j}-\frac{2}{\vartheta} \frac{c}{u_{\eta \eta}}\left(1+\operatorname{tr}\left(u^{i j}\right)+\left|D^{2} u\right|\right) \\
& \geq-c \vartheta\left(u_{11}-\chi\right)\left(\operatorname{tr}\left(u^{i j}\right)+\alpha^{2} \operatorname{tr}\left(u^{i j}\right)+\gamma^{2}\left|D^{2} u\right|\right) \\
& \quad-\frac{1}{\vartheta} \frac{c}{u_{\eta \eta}}\left(1+\operatorname{tr}\left(u^{i j}\right)+\left|D^{2} u\right|\right) .
\end{aligned}
$$

Combining this inequality with (5.10) gives

$$
\begin{aligned}
0 \geq & \left(2 \gamma-c \vartheta \gamma^{2}-\frac{c}{\vartheta(\Delta u)^{2}}-c\right) \Delta u-c(1+\alpha+\gamma) \\
& +\left(\alpha \lambda-c-c \vartheta \alpha^{2}-\frac{c}{\vartheta(\Delta u)^{2}}-\frac{c}{\Delta u}\right) \operatorname{tr}\left(u^{i j}\right) .
\end{aligned}
$$

We fix $\gamma, \alpha=\alpha(\gamma)$ sufficiently large, and finally $\vartheta=\vartheta(\gamma, \alpha)$ sufficiently small. This implies an upper bound on $u_{11}$. Note that as before, first we have fixed $\gamma$ and then $\alpha$. 
Now, it remains to consider the case

$$
(1-\vartheta) u_{\eta \eta} \geq\left(u_{11}-\chi\right) \text {. }
$$

We assume that we are in the nontrivial situation where

$$
\left(1-\frac{\vartheta}{2}\right) u_{\eta \eta} \geq u_{11} \text {. }
$$

Set

$$
\bar{\Omega} \times S^{n-1} \times[0, T] \ni(x, \xi, t) \mapsto \widetilde{W}(x, \xi, t)=\frac{e^{\alpha(\psi-u)+\gamma|D u|^{2}}\left(u_{\xi \xi}-\chi\right)}{1+c_{1}\left\langle\nu, e_{1}\right\rangle^{2}-\frac{2\left\langle\nu, e_{1}\right\rangle\left\langle\beta^{T}, e_{1}\right\rangle}{\langle\beta, \nu\rangle}},
$$

where $\chi$ is introduced in (5.6). Assume that $\widetilde{W}$ attains its maximum at a positive time $t_{\widetilde{W}}$ at $x_{\widetilde{W}} \in \bar{\Omega}$ for a direction $\xi \in S^{n-1}$. Assume further, that $x_{\widetilde{W}} \in \partial \Omega$. If $x_{\widetilde{W}} \in \Omega$, a modification of the proof for the case, when $W$ attains its maximum in $\Omega \times(0, T]$, implies a bound for the second spatial derivatives of $u$. Using a decomposition of $\xi$ as in (3.1), we obtain for $\beta$ sufficiently $C^{0}$-close to $\nu$

$$
|\tau(\xi)|^{2} \leq 1+c\left\|\beta^{T}\right\|_{C^{0}} .
$$

As a direct consequence of this decomposition, we see that

$$
u_{\xi \xi} \leq u_{\tau(\xi) \tau(\xi)}+c .
$$

We apply (5.7) and (5.11)

$$
\begin{aligned}
\widetilde{W}\left(x_{\widetilde{W}}, \xi, t_{\widetilde{W}}\right) & \leq|\tau(\xi)|^{2} W\left(x_{w}, t_{w}\right)+c \\
& \leq\left(1+c\left\|\beta^{T}\right\|_{C^{0}}\right) W\left(x_{W}, t_{W}\right)+c \\
& \leq\left(1-\frac{\vartheta}{2}\right)\left(1+c\left\|\beta^{T}\right\|_{C^{0}}\right) \widetilde{W}\left(x_{W}, \eta, t_{W}\right)+c \\
& \leq\left(1-\frac{\vartheta}{2}\right)\left(1+c\left\|\beta^{T}\right\|_{C^{0}}\right) \widetilde{W}\left(x_{\widetilde{W}}, \xi, t_{\widetilde{W}}\right)+c,
\end{aligned}
$$

where $c=c(\alpha, \gamma)$. For $\beta^{T}$ sufficiently small, we obtain a uniform bound on $\widetilde{W}\left(x_{\widetilde{W}}, \xi, t_{\widetilde{W}}\right)$.

\section{Longtime existence and convergence.}

So far, we have obtained uniform estimates on $\dot{u}, D u$, and $D^{2} u$ as long as a smooth solution exists. For $t=0$, we enclose our initial value $u_{0}$ by translating solutions. The maximum principle implies, that $u$ will stay between the translating solutions. We obtain that

$$
-c+v^{\infty} \cdot t \leq u \leq+c+v^{\infty} \cdot t .
$$

We apply Hölder estimates for the second derivatives due to Evans, Krylov, and Safonov, as well as Schauder estimates, see [3]. Since (1.1) has no explicit $u$-dependence, we get longtime existence with uniform bounds on all higher derivatives of $u$. 
To show convergence to a translating solution, we consider $w:=u-u^{\infty}$. The following argument is similar to $[\mathbf{1}, \mathbf{5}]$. Using the mean value theorem, we see that $w$ satisfies a parabolic flow equation of the form

$$
\left\{\begin{aligned}
\dot{w} & =a^{i j} w_{i j}+b^{i} w_{i} & & \text { in } \Omega \\
w_{\beta} & =0 & & \text { on } \partial \Omega .
\end{aligned}\right.
$$

Thus, the strong maximum principle implies, that $w$ is constant or its oscillation is strictly decreasing in time. In the first case, $u$ is already a translating solution. In the second case, we wish to exclude that the oscillation is strictly decreasing but does not tend to zero. If the oscillation of $w(\cdot, t)$ tends to $\varepsilon>0$ as $t \rightarrow \infty$, we consider for $t_{i} \rightarrow \infty$

$$
u^{i}(x, t):=u\left(x, t+t_{i}\right)-v^{\infty} \cdot t_{i} .
$$

We have uniform estimates in any $C^{k}$-norm for the derivatives of $u^{i}$, and locally (in time) uniform bounds for the $C^{0}$-norm, see (6.1). Hence, a subsequence of the functions $u^{i}$ converges locally (in time) uniformly in any $C^{k}$-norm to a solution $u^{*}$ of our flow equation (1.1) that exists for all time. As the oscillation of $w=u-u^{\infty}$ is monotone in $t$, we see that the oscillation of $u^{*}-u^{\infty}$ is equal to $\varepsilon$, independent of $t$. This is a contradiction to the strong maximum principle. If the oscillation of $w$ tends to zero, we see that $u$ converges to a translating solution in $C^{0}$ as $t \rightarrow \infty$. Adding a constant to the translating solution $u^{\infty}$, we may assume that $u \rightarrow u^{\infty}$ in the $C^{0}$-norm as $t \rightarrow \infty$. Interpolation inequalities of the form

$$
\|D w\|_{C^{0}(\bar{\Omega})}^{2} \leq c(\Omega) \cdot\|w\|_{C^{0}(\bar{\Omega})} \cdot\left(\left\|D^{2} w\right\|_{C^{0}(\bar{\Omega})}+\|D w\|_{C^{0}(\bar{\Omega})}\right)
$$

for $w=u-u^{\infty}$ and its derivatives imply smooth convergence. The proof of Theorem 1.1 is complete.

\section{Appendix A. Prescribing Gauß curvature for entire graphs.}

Here, we present an application of our previous existence result on bounded domains to construct unbounded hypersurfaces with prescribed Gauß curvature. Assuming that the hypersurface is given as an entire graph, the problem is to find a solution of

$$
\frac{\operatorname{det} D^{2} u}{\left(1+|D u|^{2}\right)^{\frac{n+2}{2}}}=g(x) .
$$

Observe that this equation fits in the context of the present paper, cf. (4.1)

$$
v^{\infty}=\log \operatorname{det} D^{2} u-\log f(x, D u)
$$

by defining

$$
f(x, p)=g(x) / h(p) \quad \text { with } \quad h(p)=h(|p|)=\left(1+|p|^{2}\right)^{-\frac{n+2}{2}}
$$

and looking for translating solutions with speed $v^{\infty}=0$. 
Following an argument of Altschuler and $\mathrm{Wu}[\mathbf{1}]$, we will construct entire rotationally symmetric translating solutions from solutions on growing disktype domains. Using the graph of the lower half sphere with suitably chosen radius, it is a direct consequence of the strong maximum principle that there cannot exist an entire strictly convex translating solution for any constant value of the Gauß curvature. In the rotationally symmetric case, we have the following result:

Theorem A.1. Let $g(x)=g(|x|)$ be positive, smooth and integrable. There exists an entire strictly convex solution $u$ to (A.1) if and only if

$$
v:=\log \frac{\int_{\mathbb{R}^{n}} h(|p|) d p}{\int_{\mathbb{R}^{n}} g(|x|) d x} \geq 0 .
$$

The solution constructed here has a uniformly bounded gradient if and only if $v>0$.

We remark that the rotationally symmetric setting does also allow for a proof by reducing the problem to an ordinary differential equation.

Proof. We will use that for given constants $R>0, \rho>0$, there is a unique, strictly convex solution $\left(v^{\infty}, u^{\infty}\right)$ to the following problem:

$$
\left(*_{R, \rho}\right) \quad\left\{\begin{aligned}
e^{v^{\infty}} & =\operatorname{det} D^{2} u^{\infty} \frac{h\left(\left|D u^{\infty}\right|\right)}{g(|x|)} & & \text { in } B_{R}(0), \\
u_{\nu}^{\infty} & =-\rho & & \text { on } \partial B_{R}(0), \\
u^{\infty}(0) & =0 . & &
\end{aligned}\right.
$$

This is a direct application of Theorem 1.2. Furthermore, the uniqueness of the solution implies its rotational symmetry. The solution can also be found by imposing a second boundary value condition.

From the strict convexity, we deduce that $D u^{\infty}$ is a diffeomorphism from $B_{R}(0)$ onto $B_{\rho}(0)$. Integrating $(* R, \rho)$, we obtain

$$
e^{v^{\infty}} \int_{B_{R}} g(|x|) d x=\int_{B_{R}} \operatorname{det} D^{2} u^{\infty} h\left(\left|D u^{\infty}\right|\right) d x=\int_{B_{\rho}} h(|p|) d p
$$

which uniquely determines the speed

$$
v^{\infty}=v^{\infty}(R, \rho):=\log \frac{\int_{B_{\rho}} h(|p|) d p}{\int_{B_{R}} g(|x|) d x}
$$

as a function of the parameters $R, \rho$. Note that $v^{\infty}(R, \rho)$ is strictly decreasing in $R$ and strictly increasing in $\rho$ with $v^{\infty}(R, \rho) \rightarrow-\infty$ for $\rho \rightarrow 0$.

1. Nonexistence for $v<0$ : We argue similarly as in the aforementioned case of constant Gauß curvature. Now, we replace lower half spheres by suitably constructed solutions on finite domains with arbitrarily large gradients at the boundary. Since $v<0$, there exists a unique $\hat{R}$ such that $\int_{B_{\hat{R}}} g(|x|) d x=$ $\int_{\mathbb{R}^{n}} h(|p|) d p$. Assuming that we have an entire solution $u$ of $(\mathrm{A} .1)$, there is a 
$\rho>0$ such that $|D u(x)|<\rho / 2$ for all $|x|<\hat{R}$. Now, take the unique $R<\hat{R}$ satisfying

$$
\int_{B_{R}} g(|x|) d x=\int_{B_{\rho}} h(|p|) d p
$$

and consider the solution $\left(v^{\infty}, u^{\infty}\right)$ of $\left(*_{R, \rho}\right)$. By definition, we know that $v^{\infty}=v^{\infty}(R, \rho)=0$, hence, $u^{\infty}$ solves Equation (A.1) in $B_{R}(0)$. Furthermore, the Neumann boundary condition and our choice of $\rho$ yield $\left|D u^{\infty}\right|>$ $|D u|$ in a neighborhood of $\partial B_{R}(0)$. Thus, there is a translate $u^{\infty}+m$, $m \in \mathbb{R}$, which is strictly greater than $u$. Now we shift back until the graphs touch first at a point $x \in B_{R}(0)$. By the strong maximum principle, this is impossible as $u$ and $u^{\infty}$ solve the same elliptic equation in $B_{R}(0)$.

2. Existence for $v \geq 0$ : We construct our solution by choosing a sequence of increasing radii $R_{k}$ tending to $\infty$. By the monotonicity properties of the function $v^{\infty}(R, \rho)$, we can find for each $R>0$ a unique $\rho_{R}$ such that $v^{\infty}\left(R, \rho_{R}\right)=0$. We remark that $\rho_{R}$ is an increasing sequence. Again, Theorem 1.2 gives a unique smooth rotationally symmetric solution $u_{R}$ to $\left(*_{R, \rho_{R}}\right)$, which is defined on $B_{R}(0)$ and satisfies $v^{\infty}=0$. Note that for fixed $R$, the speed $v^{\infty}$ and the normal derivative at the boundary, $-\rho_{R}$, are uniquely related. Hence, the solution $u_{R}$ must coincide on smaller balls $B_{R^{\prime}}(0), R^{\prime}<R$, with the previous solutions $u_{R^{\prime}}$ to $\left(*_{R^{\prime}, \rho_{R^{\prime}}}\right)$. Therefore, as $R$ tends to $\infty$, the sequence $\left\{u_{R}\right\}$ will converge uniformly on compact sets to a limit $u$, defined on all of $\mathbb{R}^{n}$. Clearly, $u$ is a rotationally symmetric solution to (A.1). Observe that the sequence $\rho_{R}$ will diverge in the case $v=0$, whereas it stays bounded for $v>0$. This proves the boundedness of $|D u|$ in the latter case.

Proceeding as in the existence part of the proof, we get easily that nonintegrable $g(x)$ also allow for solutions provided that $h(p)$ is non-integrable too.

This observation can be extended to the function $h(p)$ arising in the equation of prescribed Gauß curvature in Minkowski space

$$
\frac{\operatorname{det} D^{2} u}{\left(1-|D u|^{2}\right)^{\frac{n+2}{2}}}=g(x) \text {. }
$$

Hence, $h(p)=h(|p|)=\left(1-|p|^{2}\right)^{-\frac{n+2}{2}}$, which is not integrable on $B_{1}(0)$.

Theorem A.2. For all positive and smooth functions $g(x)=g(|x|)$, there exists an entire strictly convex solution $u$ to (A.2) satisfying $|D u|<1$. Moreover, for a solution constructed here, $|D u| \leq 1-\varepsilon, \varepsilon>0$, if and only if $g$ is integrable. 
Proof. We proceed similarly as in the proof of Theorem A.1 and use the notation introduced there. Here, the speed function $v^{\infty}(R, \rho)$ is only defined for $\rho<1$. But still, $v^{\infty}(R, \rho)$ is strictly decreasing in $R$ and strictly increasing in $\rho$. In addition, $v^{\infty}(R, \rho) \rightarrow-\infty$ for $\rho \rightarrow 0$ and $v^{\infty}(R, \rho) \rightarrow \infty$ for $\rho \rightarrow 1$. As in part 2 of the proof of Theorem A.1, we can find for any $R>0$ a unique $\rho_{R} \in(0,1)$ satisfying $v^{\infty}\left(R, \rho_{R}\right)=0$. Since $\rho_{R}<1$, we can choose a smooth function $\widetilde{h}(p)$, defined on $\mathbb{R}^{n}$, such that $h(p)=\widetilde{h}(p)$ for all $|p| \leq \rho_{R}$. Again, Theorem 1.2 gives a unique smooth convex rotationally symmetric solution $u_{R}$ to $\left(*_{R, \rho_{R}}\right)$ with $h$ replaced by $\widetilde{h}$, which is defined on $B_{R}(0)$ and satisfies $v^{\infty}=0$. We remark that the convexity of $u$ implies that $|D u| \leq \rho_{R}$ on $B_{R}(0)$. Thus, $u$ also solves the elliptic equation, if we replace $\widetilde{h}$ by the original $h$. Again, for $R>R^{\prime}, u_{R}$ will coincide with solutions $u_{R^{\prime}}$ obtained on smaller balls $B_{R^{\prime}}(0)$. Hence, for $R$ tending to infinity, $u_{R}$ converges to an entire solution $u$ of (A.2). In the present case, the sequence $\rho_{R}$ stays uniformly bounded away from 1 if $\int_{\mathbb{R}^{n}} g(|x|) d x<\infty$, whereas $\rho_{R}$ converges to 1 if $g$ is non-integrable.

In the general case without rotational symmetry, a theorem corresponding to Theorem 1.1 in Minkowski space can be obtained easily from the techniques of this paper, provided that there holds a uniform a priori bound of the form $|D u|<1-\varepsilon, \varepsilon>0$.

\section{Appendix B. Illustrations.}

To illustrate the convergence of solutions, we investigate numerically the flow equation

$$
\left\{\begin{aligned}
\dot{u} & =\log \operatorname{det} D^{2} u & & \text { in } \Omega \times[0, \infty), \\
u_{\nu}(\cdot, t) & =\left(u_{0}\right)_{\nu} & & \text { on } \partial \Omega, t>0, \\
u(\cdot, 0) & =u_{0} & & \text { in } \Omega
\end{aligned}\right.
$$

on the ellipsoidal domain $\Omega=\left\{(x, y) \in \mathbb{R}^{2}: 1.1 \cdot\left(x^{2}+(2 y)^{2}\right)<1\right\}$, where $u_{0}(x, y)=1.5 x^{2}+y^{2}-0.1 y^{4}$.

The numerical integration has been carried out on a $200 \times 100$ grid corresponding to $[-1,1] \times[-0.5,0.5] \in \mathbb{R}^{2}$. Let $\Omega_{0}$ consist of all grid points contained in $\Omega$, and $\partial \Omega_{0}$ denotes those grid points not contained in $\Omega_{0}$ such that one of the nearest neighbors belongs to $\Omega_{0}$. For simplicity, we keep the same notation for the discretized quantities.

We use an explicit scheme for time integration. The boundary condition is implemented as follows: For all $x_{0} \in \partial \Omega$ let $y_{0}:=x_{0}+\nu\left(x_{0}\right) \cdot \tau_{0}$, where $\nu\left(x_{0}\right)$ is the normalized negative gradient of $x^{2}+(2 y)^{2}$ and $\tau_{0}=\inf \{\tau$ : $x_{0}+\nu\left(x_{0}\right) \cdot \tau \in$ convex hull $\left.\left(\Omega_{0}\right)\right\}$. We set $u\left(x_{0}\right):=u_{0}\left(x_{0}\right)-u_{0}\left(y_{0}\right)-u\left(y_{0}\right)$. Here, $u\left(y_{0}\right)$ is obtained by linear interpolation. 


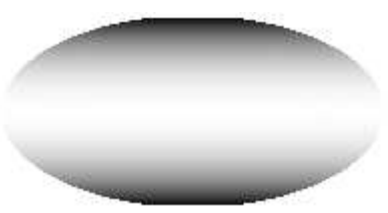

(a) $t=0.0$

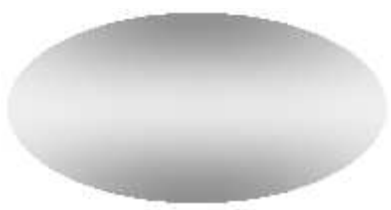

(b) $t=0.1$

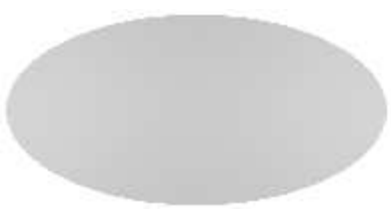

(c) $t=0.5$

Figure 2. Time evolution on an ellipsoidal domain.

Figure 2 shows a gray-scale plot of the velocity $\dot{u}$ at different times. It can be seen that the velocity tends to a constant, reflecting the convergence of $u$ to a translating solution.

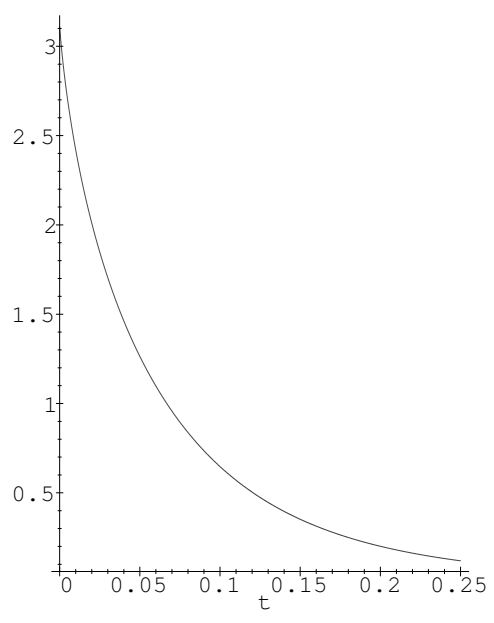

(a) $\delta(t)$

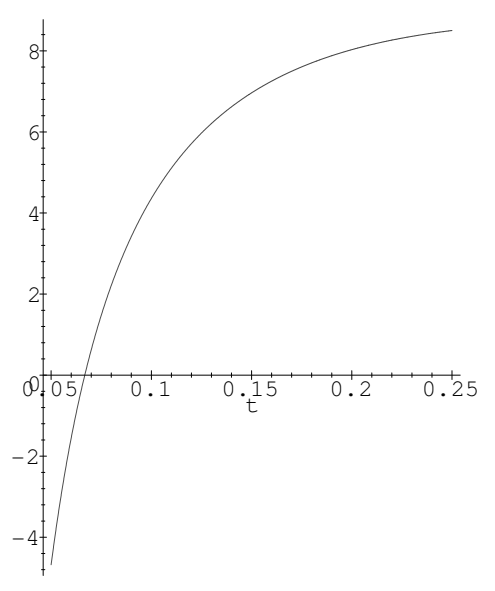

(b) $\frac{-1}{t} \log \delta(t)$

Figure 3. Convergence to constant velocity.

In Figure 3a, we show the decay of $\delta(t)=\|\dot{u}(t)-\bar{v}(t)\|_{L^{2}(\Omega)}^{2}$, where $\bar{v}(t)=$ $\frac{1}{|\Omega|} \int \dot{u}(x, t) d x$ is the mean velocity. The expected exponential convergence can be seen from Figure $3 b$. Here, we plot the exponential rate $\frac{-1}{t} \log \delta(t)$, which saturates nicely for larger times.

Acknowledgements. We wish to thank Jürgen Jost, Stefan Müller and the Max Planck Institute for Mathematics in the Sciences for their hospitality. The first author also wishes to acknowledge that this paper was finished while he was at Harvard University, supported by the Humboldt foundation. 


\section{References}

[1] S.J. Altschuler and L.F. Wu, Translating surfaces of the non-parametric mean curvature flow with prescribed contact angle, Calc. Var. Partial Differential Equations, 2 (1994), 101-111, MR 97b:58032, Zbl 0812.35063.

[2] G. Huisken, Nonparametric mean curvature evolution with boundary conditions, J. Differential Equations, 77 (1989), 369-378, MR 90g:35050, Zbl 0686.34013.

[3] G.M. Lieberman, Second Order Parabolic Differential Equations, World Scientific Publishing Co., Inc., River Edge, NJ, 1996, MR 98k:35003, Zbl 0884.35001.

[4] P.-L. Lions, N.S. Trudinger and J.I.E. Urbas, The Neumann problem for equations of Monge-Ampère type, Comm. Pure Appl. Math., 39 (1986), 539-563, MR 87j:35114, Zbl 0604.35027.

[5] O.C. Schnürer, Translating solutions to the second boundary value problem for curvature flows, Manuscripta Math., 108 (2002), 319-347, MR 2003f:53120.

[6] O.C. Schnürer and K. Smoczyk, Neumann and second boundary value problems for Hessian and Gauß curvature flows, Ann. Inst. H. Poincaré Anal. Non Linéaire, 20 (2003), 1043-1073.

[7] J. Urbas, Oblique boundary value problems for equations of Monge-Ampère type, Calc. Var. Partial Differential Equations, 7 (1998), 19-39, MR 99h:35068, Zbl 0912.35068.

[8] _ Global Hölder estimates for equations of Monge-Ampère type, Invent. Math., 91 (1988), 1-29, MR 89b:35047, Zbl 0674.35026.

[9] _ The equation of prescribed Gauss curvature without boundary conditions, J. Differential Geom., 20 (1984), 311-327, MR 87a:53099, Zbl 0566.53013.

Received August 26, 2002.

Max Planck Institute for Mathematics in the Sciences

INSELSTR. 22-26, 04103 LEIPZIG

GERMANY

E-mail address: Oliver.Schnuerer@mis.mpg.de

Department of Mathematics and Computer Science

Free University Berlin

ARNimallee 2-6

14195 BERLIN

Germany

Max Planck Institute for Mathematics in the Sciences

INSELSTR. 22-26, 04103 LEIPZIG

GERMANY

E-mail address: Hartmut.Schwetlick@mis.mpg.de 